\title{
Parameterizable Object
}

National Cancer Institute

\section{Source}

National Cancer Institute. Parameterizable Object. NCI Thesaurus. Code C44176.

The Parameterizable interface encapsulates the association of Parameters with

ParameterValues. (caMAGE) 